\title{
Primary infertility associated with schitosoma mansoni: a case report from the Jos plateau, north central Nigeria
}

\author{
Adisa $\mathrm{J}^{1}$, Egbujo $\mathrm{EM}^{2}$, Yahaya BA ${ }^{3}$, Echejoh $\mathrm{G}^{4}$
}

1. University of Jos, Medical laboratory Science, Nigeria

2. Histopathology Laboratory Services, Jos, Nigeria

3. Jos University Teaching Hospital, Histopathology, Nigeria

4. Jos University Teaching Hospital, Histopathology, Nigeria

\begin{abstract}
Testicular schistosomiasis caused by Schistosoma mansoni is very rare and more so when associated with primary infertility. A 40 years old man from the Jos Plateau, North Central Nigeria presented with primary infertility after ten years of marriage. Sperm count revealed oligospermia and he also complained of inability to sustain erection. Testicular biopsy revealed several ova of Schistosoma mansoni in the connective tissue of the testes. Cases of infertility in endemic areas especially when there is no supply of potable water should raise suspicion of schistosomiasis among other pathologies and possible hormonal disturbances when the testes are involved.
\end{abstract}

Key words: Schistosomiasis, testes, infertility

African Health Sciences 2012; (4): 563 - 565 http://dx.doi.org/10.4314/ahs.v12i4.26

\section{Introduction}

Schistosomiasis is a parasitic disease caused by blood flukes (trematodes) of the genus Schistosoma. After malaria and intestinal helminthiasis, schistosomiasis is the third most devastating tropical disease in the world, being a major source of morbidity and mortality for developing countries in Africa, South America, the Caribbean, the Middle East, and Asia ${ }^{1}$.

More than 207 million people, $85 \%$ of who live in Africa, are infected with schistosomiasis ${ }^{1}$ and an estimated 700 million people are at risk of infection in 76 countries where the disease is considered endemic, as their agricultural work, domestic chores, and recreational activities expose them to infested water $^{1,2}$.

Sometimes referred to as bilharzias, bilharziasis, or snail fever, schistosomiasis was discovered by Theodore Bilharz, a German surgeon working in Cairo, who first identified the etiological agent Schistosoma hematobium in $1851^{3}$.

Testicular schistosomiasis caused by $S$. beamatobium is relatively more common than that caused by S. mansoni. It is presumed that in the case

\footnotetext{
*Corresponding author:

James Adisa

University of Jos

Medical laboratory Science

Nigeria

Email: adisawuraola@yahoo.com

adisawuraola@yahoo.com
}

of S. mansoni it may be due to migration of eggs through venous channels between the internal spermatic and mesenteric veins ${ }^{4}$. There are few reports of testicular schistosomiasis described in literature owing to the rarity of this entity in this organ. It usually mimics a malignant lesion presenting with painless small solid nodule ${ }^{5,6}$. It has also been associated with erectile dysfunction and infertility in endemic areas ${ }^{6,7,8}$. Severe manifestation of schistosomiasis can cause intense granulomatous epididymitis and inhibition of spermatogenesis causing male infertility?.

Fertility can also be impaired through any of 4 basic mechanisms: direct gonadotoxic effects, alteration of the hypothalamic-pituitary-gonadal (HPG) axis, direct effects on ejaculation and/or erectile function, and direct effects on libido ${ }^{8}$

\section{Case report}

A 40 years old man presented with a complaint of inability to impregnate his wife after 10 years of marriage. He had to seek medical attention because he wanted to remarry. This patient is from the low lands of the Jos Plateau, located in North Central region of Nigeria which is endemic for schitosomiasis. The area is swampy and well suited for yam and rice cultivation both of which he was engaged in. On further probing, the patient reported that he also constantly passed through a river on his way to his farm and back. 
The patient came to the hospital because his first wife left him a few years ago and now he wanted to remarry. He was also worried because he noticed that he could no longer sustain erection. He could however not specify when erectile dysfunction became a serious issue but the departure of his wife may not be unconnected with this development. Pretesticular causes related to male infertility revealed that the patient is a moderate consumer of both refined and locally brewed alcohol and did not misuse drugs generally nor use same to boost his sexual drive. He was also not involved in strenuous bicycle riding that could affect the optimum temperature for spermatogenesis. On examination however, no nodule was observed in his testes. Sperm count conducted revealed oligospermia in two separate counts with each count less than $20 \mathrm{x}$ $10^{6} / \mathrm{ml}$.

A testicular biopsy was obtained from the left testis and histopathology revealed ova of Schistosoma mansoni with their lateral spine in the connective tissue with lymphocytic infiltrate (figure 1).

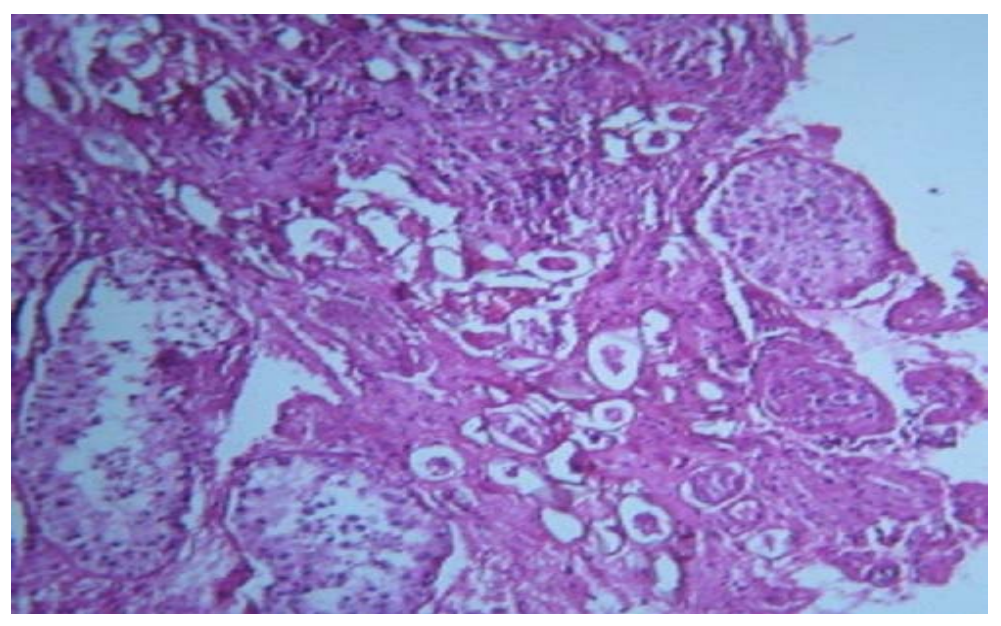

Figure 1: Histologic section of testis showing several seminiferous tubules around the connective tissue in which are trapped ova of Schistosoma mansoni (arrows) and infilerate of lymphocytes. Heamatoxylin and Eosin Method (Magnification- X 150)

The infection did not in any way affect the seminiferous tubules of the testes. The patient was treated with Praziquantel and Vitamin C. Determination of testosterone level was not considered necessary again, since in the first instance hormonal cause was ruled out before a testicular biopsy was undertaken. At the time of this publication, the patient is excited with improvement he has observed with his erection and the prospect of a sexually fulfilling and productive marital life, suggesting the association of the condition with schistosomiasis.

This patient volunteered all personal information used and gave an informed consent for this case study to be published.

\section{Discussion}

Testicular schistosomiasis due to $S$. mansoni is very rare especially in this area and infertility resulting from this is also not a common occurrence. The case of this patient is instructive to clinicians who should be more aware of the possibility of schistosomiasis especially among rural dwellers and its possible contribution to subfertility in endemic areas.

The reported oligospermia with chronic inflammatory lymphocytic infiltrate in the connective tissue of the testes is caused by trapped eggs which also cause other major damages ${ }^{11}$. This could also cause occlusion of the spermatic venous plexus by ova and subsequent granuloma formation may result in testicular infarction. A similar case was reported by Suresh Kini et al in which they reported the case of a patient with testicular schistosomiasis. The patient also had significant loss of libido during his illness and was given three courses of praziquantel to treat the schistosoma infection. The seminal analysis demonstrated persistent azoospermia with normal levels gonadotropins and normal testosterone suggestive of obstructive azoospermia? It is our opinion that the case of infertility in our case study is also caused by obstruction due to granuloma formation around the numerous eggs trapped in the testes. 
Experimental schistosomiasis ${ }^{12}$ has revealed that decreased testosterone levels in testicular schistisomiasiscould lead to oligospermia as often observed in infertile men. It is well known that testosterone stimulates the synthesis of specific epididymal proteins essential for post testicular sperm differentiation, capacitation and acquisition of fecundity ${ }^{13}$. A low level of testosterone would not be adequate for epididymal maturation of spermatozoa. In infected mice, it has been reported that spermatozoa from the seminiferous tubules could not acquire progressive motility and passage through the epididymis ${ }^{6}$. Treatment of testicular schistosomiasis should therefore take into cognisance the effect of the infection on relevant hormonal changes.

Also known as bilharziasis, is a parasitic disease that can lead to chronic ill health. Causal agents of the disease are fluke worms (schistosomes). A person infected with schistosomiasis expels eggs in their faeces or urine. On contact with water, the eggs hatch and release larvae called miracidia. If the miracidia find the right type of fresh water snail they use it to multiply in several cycles, eventually producing thousands of new parasites, called cercariae, which the snail then releases into the surrounding water. Humans become infected when they come into contact with contaminated water (during wading, swimming, washing). Cercariae penetrate the skin and make their way into the person's bloodstream. Over the next 30-45 days, the parasite transforms itself into a long worm-either male or female-which makes its way to the intestine or bladder. In their final location, the female lays between 200 and 2000 eggs per day over an average of 5 years. Only half of the eggs produced are excreted in the faeces or urine. The rest become trapped in the body tissues and this causes major damage ${ }^{11}$.

Urogenital schistosomiasis, caused by schistosoma haematobium, is a major health problem in many parts of Africa. Infertility may develop in males with schistosoma haematobium infection of the genital organs. Occlusion of the spermatic venous plexus by ova and subsequent granuloma formation may result in testicular infarction. Infertility may be prevented by treatment with praziquantel and early diagnosis of schistosomiasis is essential to avoid the development of infertility ${ }^{14}$.

\section{Conclusion}

Schistosomiasis is an important cause for sub-fertility in endemic areas and early diagnosis and treatment with praziquantel can potentially prevent infertility. Complications from chronic schistosomiasis are common, they can be devastating and irreversible.

\section{References}

1. World Health Organization. Weekly epidemiological record. 2010;18 (85): 157-164

2. World Health Organization (Feburary 2010) Scistosomiasis, Fact Sheet No 115.

3. Nawal M. Nour Schistosomiasis: Health Effects on Women. Reviews in Obstetrics and Gynecology 2010; 3: 28-32.

4. Lopes RI, Lopes RN, Leite KR, Prando D, Lopes RN. Testicular schistosomiasis caused by Schistosoma mansoni: A Case Report from Brazil. The Brazilian Journal of Infectious Diseases. 2007; 11(5):523-524.

5. Wedel PG, Jess P, Testicular schistosomiasis simulating malignancy. Scand J Urol Nephrol. 1991; 25: 237-8.

6. Neto NM, Grando PSJ, Moreira HA. Testicular schistosomiasis mimicking tumour. Int. Braz J Urol. 2004; 30(6): 502-503.

7. Josiah SD, Manko PL. The Effect of Schistosoma mansoni cercaria infection and Treatment with Niridazole on Testicular Histology of the mice. African Journal of Clinical Experimental Microbiology. 2003; 4(1): 2-5.

8. Jatsa HB, Kamtchouing P, Takougang ISD. Testicular Dysfunction in BALB C mice with Schistosoma intercalatum bilharziasis. Asian J Andrology. 2002; 4:143-147.

9. Kini S, Dayoub N, Raja A, Pickering S, Thong J. Unusual association of diseases/symptoms Schistosomiasis-induced male infertility Case Reports : BMJ 2009; 10.1136/bcr.01.1481

10. Nudell DM, Monoski MM, Lipshultz LI. (2002) Common medications and drugs: how they affect male fertility. Urol Clin North Am.; 29: 965-973.

11. World Health Organization. Useful Information on Schistosomiasis. WHO Fact sheet. 1999

12. Kuntz RE, Mc Coullough B, Moore JA, Huang T.C. Experimental Infection with Schistosoma intercalatum (Fisher, 1934) in Chinpanzee (Pan troglodytes) and gibbon (hylobates lar). Am J Trop Med Hyg 1978; 27: 632-4.

13. Cunningham GR, Huckins C. Persistence of complete spermatogenesis in the presence of low intratesticular concentration of testosterone. Endocrinology. 1979; 105: 177-87.

14. Toresi J, Sheori H, Ryan N, et al. Usefulness of semen microscopy in the diagnosis of a difficult case of schistoma haematobium infection in a returned traveller. J Travel Med 1997; 44: 46-7. 